Korean J. Math. 21 (2013), No. 3, pp. 285-292

http://dx.doi.org/10.11568/kjm.2013.21.3.285

\title{
ANALYTIC CONTINUATION OF GENERALIZED NON-HOLOMORPHIC EISENSTEIN SERIES
}

\author{
SUNG-GEUN LIM
}

\begin{abstract}
B. C. Berndt computed the Fourier series of a class of generalized Eisenstein series, which gives an analytic continuation to the generalized Eisenstein series. In this paper, continuing his work, we consider generalized non-holomorphic Eisenstein series and give an analytic continuation to the $s$-plane.
\end{abstract}

\section{Introduction}

B. C. Berndt [3] proved transformation formulae for a more general class of Eisenstein series which is defined by

$$
G\left(z, s ; r_{1}, r_{2}, h_{1}, h_{2}\right):=\sum_{m, n=-\infty}^{\infty} \frac{e^{2 \pi i\left(m h_{1}+n h_{2}\right)}}{\left(\left(m+r_{1}\right) \tau+n+r_{2}\right)^{s}},
$$

where the dash ' means $(m, n) \neq\left(-r_{1},-r_{2}\right)$, Im $z>0, r_{1}, r_{2}, h_{1}, h_{2}$ are real and Re $s>2$. He [4] has converted those formulae into transformation formulae for a large class of series including the Dedekind eta function, which give $G\left(z, s ; r_{1}, r_{2}, h_{1}, h_{2}\right)$ an analytic continuation to all values of $s \in \mathbb{C}$. Using these transformation formulae, he has established a lot of infinite series identities which include many infinite series identities by Ramanujan or by other authors. In addition, these

Received July 4, 2013. Revised August 2, 2013. Accepted August 7, 2013.

2010 Mathematics Subject Classification: Primary 34A25; Secondry 11M36.

Key words and phrases: Analytic continuation, non-holomorphic Eisenstein series.

(c) The Kangwon-Kyungki Mathematical Society, 2013.

This is an Open Access article distributed under the terms of the Creative commons Attribution Non-Commercial License (http://creativecommons.org/licenses/by -nc/3.0/) which permits unrestricted non-commercial use, distribution and reproduction in any medium, provided the original work is properly cited. 
transformation formulae have been applied to obtain transformation formulae for theta functions and reciprocities of modified Dedekind sums. Recently, the author could derive new proofs of three modular transformations which had been given by J. Lehner, K. Mahlburg, Y. Yang, respectively [7].

In this paper, we consider a class of generalized non-holomorphic Eisenstein series. Using generalized Lipschitz summation formula [9] and applying Berndt's method [2], we convert generalized non-holomorphic Eisenstein series into certain series with confluent hypergeometric functions of the second kind $[6,11]$, which give an analytic continuation under some condition to the entire $s$-plane. It is worthy of notice that the confluent hypergeometric functions of the first and the second kind are used to obtain analytic continuation of each case and properties of the hypergeometric function ${ }_{2} F_{1}(a, b ; c ; z)$ are essential to obtain transformation formulae which are defined for all values of $s$ in $\mathbb{C}[6,10,11]$.

\section{Notations}

For a complex $w$, we choose the branch of the argument defined by $-\pi \leq \arg w<\pi$. Let $\lambda$ denote the characteristic function of the integers and let $e(w)=e^{2 \pi i w}$. For every complex $\tau, V \tau=V(\tau)=\frac{a \tau+b}{c \tau+d}$ always denotes a modular transformation with $c>0$. Let $r=\left(r_{1}, r_{2}\right)$ and $h=\left(h_{1}, h_{2}\right)$ denote real vectors, and define the associated vectors $R$ and $H$ by

$$
R=\left(R_{1}, R_{2}\right)=\left(a r_{1}+c r_{2}, b r_{1}+d r_{2}\right)
$$

and

$$
H=\left(H_{1}, H_{2}\right)=\left(d h_{1}-b h_{2},-c h_{1}+a h_{2}\right) .
$$

For a real number $x,[x]$ denotes the greatest integer less than or equal to $x$ and $\{x\}:=x-[x]$. For real $x, \alpha$ and Re $s>1$, let

$$
\psi(x, \alpha, s):=\sum_{n+\alpha>0} \frac{e(n x)}{(n+\alpha)^{s}} .
$$

We see that $\psi(x, \alpha, s)$ can be analytically continued to the entire $s$ plane [1]. The confluent hypergeometric function of the second kind $U(\alpha, \beta, z)$ is defined to be

$$
U(\alpha, \beta, z):=\frac{\Gamma(1-\beta)}{\Gamma(1+\alpha-\beta)}{ }_{1} F_{1}(\alpha ; \beta ; z)
$$




$$
+\frac{\Gamma(\beta-1)}{\Gamma(\alpha)} z_{1}^{1-\beta} F_{1}(1+\alpha-\beta ; 2-\beta ; z)
$$

where

$$
{ }_{1} F_{1}(\alpha ; \beta ; z):=\sum_{n=0}^{\infty} \frac{(\alpha)_{n}}{(\beta)_{n} n !} z^{n}
$$

and $(x)_{n}$ denotes the rising factorial defined by

$$
(x)_{n}:=x(x+1) \cdots(x+n-1) \text { for } n>0,(x)_{0}:=1 .
$$

By the functional equation of gamma functions, we see

$$
\begin{aligned}
& U(\alpha, \beta, z) \\
& =\frac{\pi}{\sin \pi \beta}\left(\frac{{ }_{1} F_{1}(\alpha ; \beta ; z)}{\Gamma(\beta) \Gamma(1+\alpha-\beta)}-z^{1-\beta} \frac{{ }_{1} F_{1}(1+\alpha-\beta ; 2-\beta ; z)}{\Gamma(2-\beta) \Gamma(\alpha)}\right) .
\end{aligned}
$$

Then $U(\alpha, \beta, z)$ can be analytically continued to all values of $\alpha, \beta$ and $z$ real or complex, even when $\beta$ is zero or a negative integer [10], where $z$ is in the plane cut along $[-\infty, 0]$. We also have an integral expression for $U(\alpha, \beta, z)$, which is [10], for $\operatorname{Re} \alpha>0$, $\operatorname{Re} z>0$,

$$
U(\alpha, \beta, z)=\frac{1}{\Gamma(\alpha)} \int_{0}^{\infty} t^{\alpha-1}(1+t)^{\beta-\alpha-1} e^{-z t} d t .
$$

Thus the right hand side of (2.1) can be analytically continued to all $\alpha, \beta$ and $z$, where $|\arg z|<\pi$. The function $U(\alpha, \beta, z)$ has asymptotic series $[6]$

$$
U(\alpha, \beta, z)=z^{-\alpha}\left(1+O\left(\frac{1}{z}\right)\right),|z| \rightarrow \infty,
$$

where $|\arg z|<\pi$.

\section{Generalized non-holomorphic Eisenstein series}

Let $\mathbb{H}=\{\tau \in \mathbb{C} \mid \operatorname{Im}(\tau)>0\}$, the upper half-plane.

Definition 3.1. Let $\tau \in \mathbb{H}$ and $s, s_{1} \in \mathbb{C}$. For Re $s>2$, the generalized non-holomorphic Eisenstein series $\mathbf{G}\left(\tau, \bar{\tau}, s, s_{1} ; r, h\right)$ is defined to 
be

$$
\begin{aligned}
\mathbf{G}\left(\tau, \bar{\tau}, s, s_{1} ; r, h\right) & := \\
\sum_{m, n=-\infty}^{\infty} & \frac{e\left(m h_{1}+n h_{2}\right)}{\left(\left(m+r_{1}\right) \tau+n+r_{2}\right)^{s_{1}}\left(\left(m+r_{1}\right) \bar{\tau}+n+r_{2}\right)^{s-s_{1}}},
\end{aligned}
$$

where the dash' means $(m, n) \neq\left(-r_{1},-r_{2}\right)$.

For $\tau \in \mathbb{H}$ and arbitrary $s_{1}, s \in \mathbb{C}$, define

$$
\begin{gathered}
\mathcal{A}\left(\tau, s, s_{1} ; r, h\right):=\sum_{\substack{m+r_{1}>0 \\
\cdot U\left(s-h_{2}>0\right.}} \frac{e\left(m h_{1}+\left(\left(m+r_{1}\right) \tau+r_{2}\right)\left(n-h_{2}\right)\right)}{\left(n-h_{2}\right)^{1-s}} \\
\left.\cdot 4 \pi\left(m+r_{1}\right)\left(n-h_{2}\right) \operatorname{Im}(\tau)\right)
\end{gathered}
$$

and

$$
\begin{gathered}
\overline{\mathcal{A}}\left(\tau, s, s_{1} ; r, h\right):=\sum_{\substack{m+r_{1}>0 \\
\cdot U\left(s_{1} ; s ; 4 \pi\left(m+h_{2}>0\right.\right.}} \frac{e\left(m h_{1}-\left(\left(m+r_{1}\right) \bar{\tau}+r_{2}\right)\left(n+h_{2}\right)\right)}{\left(n+h_{2}\right)^{1-s}} \\
\left.\left.\qquad h_{2}\right) \operatorname{Im}(\tau)\right) .
\end{gathered}
$$

By (2.2), two functions $\mathcal{A}\left(\tau, s, s_{1} ; r, h\right)$ and $\overline{\mathcal{A}}\left(\tau, s, s_{1} ; r, h\right)$ are well-defined for all $s_{1}, s \in \mathbb{C}$. Let

$$
\mathcal{H}\left(\tau, s, s_{1} ; r, h\right):=\mathcal{A}\left(\tau, s, s_{1} ; r, h\right)+e\left(\frac{s}{2}\right) \mathcal{A}\left(\tau, s, s_{1} ;-r,-h\right)
$$

and

$$
\overline{\mathcal{H}}\left(\tau, s, s_{1} ; r, h\right):=\overline{\mathcal{A}}\left(\tau, s, s_{1} ; r, h\right)+e\left(\frac{s}{2}\right) \overline{\mathcal{A}}\left(\tau, s, s_{1} ;-r,-h\right) .
$$

Let

$$
\begin{aligned}
\mathbf{H}\left(\tau, \bar{\tau}, s, s_{1} ; r, h\right):= & \frac{1}{\Gamma\left(s_{1}\right)} \mathcal{H}\left(\tau, s, s_{1} ; r, h\right)+\frac{1}{\Gamma\left(s-s_{1}\right)} \overline{\mathcal{H}}\left(\tau, s, s_{1} ; r, h\right) .
\end{aligned}
$$

Then the function $\mathbf{H}\left(\tau, \bar{\tau}, s, s_{1} ; r, h\right)$ is well-defined for all $s_{1}, s \in \mathbb{C}$. We find a relation between $\mathbf{G}\left(\tau, \bar{\tau}, s, s_{1} ; r, h\right)$ and $\mathbf{H}\left(\tau, \bar{\tau}, s, s_{1} ; r, h\right)$.

Theorem 3.2. If $\tau \in \mathbb{H}$ and $\operatorname{Re} s>2$, then

$$
\begin{aligned}
\mathbf{G}\left(\tau, \bar{\tau}, s, s_{1}\right. & ; r, h)=\lambda\left(r_{1}\right) e\left(-r_{1} h_{1}\right) \Psi\left(h_{2}, r_{2}, s\right) \\
& +\lambda\left(h_{2}\right) \frac{(2 \pi)^{s}(-i)^{-s+2 s_{1}}}{(4 \pi \operatorname{Im} \tau)^{s-1}} \frac{\Gamma(s-1)}{\Gamma\left(s_{1}\right) \Gamma\left(s-s_{1}\right)} \Psi_{-1}\left(h_{1}, r_{1}, s\right) \\
& +(2 \pi)^{s}(-i)^{-s+2 s_{1}} \mathbf{H}\left(\tau, \bar{\tau}, s, s_{1} ; r, h\right),
\end{aligned}
$$


where

$$
\begin{aligned}
& \Psi(\alpha, \beta, s):=\psi(\alpha, \beta, s)+e\left(\frac{s}{2}\right) \psi(-\alpha,-\beta, s), \\
& \Psi_{-1}(\alpha, \beta, s):=\psi(\alpha, \beta, s-1)+e\left(\frac{s}{2}\right) \psi(-\alpha,-\beta, s-1) .
\end{aligned}
$$

Proof. For $\tau \in \mathbb{H}$ and $\operatorname{Re} s>2$,

$$
\begin{aligned}
\mathbf{G}\left(\tau, \bar{\tau}, s, s_{1} ; r, h\right)= & \lambda\left(r_{1}\right) e\left(-r_{1} h_{1}\right) \sum_{n=-\infty}^{\infty} \frac{e\left(n h_{2}\right)}{\left(n+r_{2}\right)^{s}} \\
+ & \left(\sum_{m<-r_{1}} \sum_{n=-\infty}^{\infty}+\sum_{m>-r_{1}} \sum_{n=-\infty}^{\infty}\right) \frac{e\left(m h_{1}+n h_{2}\right)}{\left(\left(m+r_{1}\right) \tau+n+r_{2}\right)^{s_{1}}} \\
& \cdot \frac{1}{\left(\left(m+r_{1}\right) \bar{\tau}+n+r_{2}\right)^{s-s_{1}}} \\
= & : T_{1}+T_{2}+T_{3}, \text { say. }
\end{aligned}
$$

Firstly,

$$
\begin{aligned}
T_{1} & =\lambda\left(r_{1}\right) e\left(-r_{1} h_{1}\right)\left(\sum_{n>-r_{2}} \frac{e\left(n h_{2}\right)}{\left(n+r_{2}\right)^{s}}+\sum_{n<-r_{2}} \frac{e\left(n h_{2}\right)}{\left(n+r_{2}\right)^{s}}\right) \\
& =\lambda\left(r_{1}\right) e\left(-r_{1} h_{1}\right)\left(\psi\left(h_{2}, r_{2}, s\right)+e\left(\frac{s}{2}\right) \psi\left(-h_{2},-r_{2}, s\right)\right) .
\end{aligned}
$$

We shall use a generalization of the Lipschitz summation formula [9], that is, if $\alpha \in \mathbb{R}$, Re $s>1$ and $\tau=x+i y \in \mathbb{H}$, then

$$
\begin{aligned}
& \sum_{m=-\infty}^{\infty} \frac{e(-\alpha m)}{(\tau+m)^{s_{1}}(\bar{\tau}+m)^{s-s_{1}}} \\
& =\lambda(\alpha) \frac{(2 \pi)^{s}(-i)^{-s+2 s_{1}} \Gamma(s-1)(4 \pi y)^{1-s}}{\Gamma\left(s_{1}\right) \Gamma\left(s-s_{1}\right)} \\
& \quad+\frac{(2 \pi)^{s}(-i)^{-s+2 s_{1}}}{\Gamma\left(s_{1}\right)} \sum_{n>-\alpha} \frac{e((n+\alpha) \tau)}{(n+\alpha)^{1-s}} U\left(s-s_{1}, s, 4 \pi(n+\alpha) y\right) \\
& \quad+\frac{(2 \pi)^{s}(-i)^{-s+2 s_{1}}}{\Gamma\left(s-s_{1}\right)} \sum_{n>\alpha} \frac{e(-(n-\alpha) \bar{\tau})}{(n-\alpha)^{1-s}} U\left(s_{1}, s, 4 \pi(n-\alpha) y\right) .
\end{aligned}
$$

Secondly, replacing $m$ by $-m$ and then using (3.3) with $\tau$ replaced by $\left(m-r_{1}\right) \tau-r_{2}$ and $\alpha$ by $h_{2}$,

$$
T_{2}=e\left(\frac{s}{2}\right) \sum_{m>r_{1}} e\left(-m h_{1}\right)
$$




$$
\begin{gathered}
\sum_{n=-\infty}^{\infty} \frac{e\left(-n h_{2}\right)}{\left(\left(m-r_{1}\right) \tau+n-r_{2}\right)^{s_{1}}\left(\left(m-r_{1}\right) \bar{\tau}+n-r_{2}\right)^{s-s_{1}}} \\
=\lambda\left(h_{2}\right) \frac{e\left(\frac{s}{2}\right)(2 \pi)^{s}(-i)^{-s+2 s_{1}}}{(4 \pi y)^{s-1}} \frac{\Gamma(s-1)}{\Gamma\left(s_{1}\right) \Gamma\left(s-s_{1}\right)} \psi\left(-h_{1},-r_{1}, s-1\right) \\
+\frac{(2 \pi)^{s}(-i)^{-s+2 s_{1}} e\left(\frac{s}{2}\right)}{\Gamma\left(s_{1}\right)} \mathcal{A}\left(\tau, s, s_{1} ;-r,-h\right) \\
+\frac{(2 \pi)^{s}(-i)^{-s+2 s_{1}} e\left(\frac{s}{2}\right)}{\Gamma\left(s-s_{1}\right)} \overline{\mathcal{A}}\left(\tau, s, s_{1} ;-r,-h\right) .
\end{gathered}
$$

By the similar manner, applying (3.3) with $\tau$ replaced by $\left(m+r_{1}\right) \tau+r_{2}$ and $\alpha$ by $h_{2}$, we find

$$
\begin{aligned}
T_{3}= & \lambda\left(h_{2}\right) \frac{(2 \pi)^{s}(-i)^{-s+2 s_{1}}}{(4 \pi y)^{s-1}} \frac{\Gamma(s-1)}{\Gamma\left(s_{1}\right) \Gamma\left(s-s_{1}\right)} \psi\left(h_{1}, r_{1}, s-1\right) \\
& +\frac{(2 \pi)^{s}(-i)^{-s+2 s_{1}}}{\Gamma\left(s_{1}\right)} \mathcal{A}\left(\tau, s, s_{1} ; r, h\right) \\
& +\frac{(2 \pi)^{s}(-i)^{-s+2 s_{1}}}{\Gamma\left(s-s_{1}\right)} \overline{\mathcal{A}}\left(\tau, s, s_{1} ; r, h\right) .
\end{aligned}
$$

Plugging (3.2), (3.4) and (3.5) into (3.1), we complete the proof.

Remark 3.3. We see that $\Psi(\alpha, \beta, s)$ and $\Psi_{-1}(\alpha, \beta, s)$ can be analytically continued to the entire $s$-plane, and $\mathbf{H}\left(\tau, \bar{\tau}, s, s_{1} ; r, h\right)$ is an entire function of $s$. Hence $\mathbf{G}\left(\tau, \bar{\tau}, s, s_{1} ; r, h\right)$ can be analytically continued to the entire $s$-plane if $h_{2}$ is not integral or $s_{1}$ is integral. If $h_{2}$ is integral and $s_{1}$ is not integral, then $\mathbf{G}$ can be meromorphically continued to entire $s$-plane, namely, it has simple poles at integers $s \leq 1$.

Recently, M. Katsurada and T. Noda [5] found an meromorphic continuation of non-holomorphic Eisenstein series $E_{k}(s ; z)$ over the whole $s$-plane for even integer $k$, where

$$
E_{k}(s ; z):=\frac{1}{2} \sum_{\substack{m, n=-\infty \\(m, n)=1}}^{\infty}(m z+n)^{-k}|m z+n|^{-2 s},(\operatorname{Re} s>1-k / 2) .
$$

Since

$$
2 \zeta(k+2 s) E_{k}(s ; z)=\mathbf{G}(z, \bar{z}, k+2 s, k+s ; 0,0),
$$

this can be regarded as a special case of Theorem 3.2.

Finally, we derive modular transformation formulae for $\mathbf{H}\left(\tau, \bar{\tau}, s, s_{1} ; r, h\right)$. 
Theorem 3.4. Let $Q=\{\tau \in \mathbb{H} \mid \operatorname{Re} \tau>-d / c\}$ and let $s_{1} \in \mathbb{C}$. Then for $\tau \in Q$ and $s \in \mathbb{C}$, not integers $\leq 1$,

$$
\begin{aligned}
(c \tau+ & d)^{-s_{1}}(c \bar{\tau}+d)^{-s+s_{1}} \mathbf{H}\left(V \tau, V \bar{\tau}, s, s_{1} ; r, h\right)=\mathbf{H}\left(\tau, \bar{\tau}, s, s_{1} ; R, H\right) \\
+ & \lambda\left(R_{1}\right) e\left(-R_{1} H_{1}\right)(2 \pi i)^{-s} e\left(-\frac{s_{2}}{2}\right) \Psi\left(-H_{2},-R_{2}, s\right) \\
- & \lambda\left(r_{1}\right) e\left(-r_{1} h_{1}\right)(2 \pi i)^{-s} e\left(\frac{s_{1}}{2}\right)(c \tau+d)^{-s_{1}}(c \bar{\tau}+d)^{-s+s_{1}} \\
& \Psi\left(h_{2}, r_{2}, s\right) \\
+ & \lambda\left(H_{2}\right)(4 \pi \operatorname{Im}(\tau))^{1-s} \frac{\Gamma(s-1)}{\Gamma\left(s_{1}\right) \Gamma\left(s-s_{1}\right)} \Psi_{-1}\left(H_{1}, R_{1}, s\right) \\
- & \lambda\left(h_{2}\right)(4 \pi \operatorname{Im}(\tau))^{1-s} \frac{\Gamma(s-1)}{\Gamma\left(s_{1}\right) \Gamma\left(s-s_{1}\right)}(c \tau+d)^{s-s_{1}-1}(c \bar{\tau}+d)^{s_{1}-1} \\
& \Psi_{-1}\left(h_{1}, r_{1}, s\right) \\
+ & \frac{(2 \pi i)^{-s} e\left(-\frac{s_{2}}{2}\right)}{\Gamma\left(s_{1}\right) \Gamma\left(s-s_{1}\right)} \mathbf{L}\left(\tau, \bar{\tau}, s, s_{1} ; R, H\right) .
\end{aligned}
$$

Proof. Employ modular transformation formulae for $\mathbf{G}\left(\tau, \bar{\tau}, s, s_{1} ; r, h\right)$ in [8] and apply Theorem 3.2.

Acknowledgement. The author thanks B. C. Berndt for his helpful suggestions for this work.

\section{References}

[1] B. C. Berndt, Two new proofs of Lerch's functional equation, Proc. Amer. Math. Soc. 32 (1972), 403-408.

[2] B. C. Berndt, Generalized Eisenstein series and modified Dedekind sums, J. Reine. Angew. Math. 272 (1975), 182-193.

[3] B. C. Berndt, Modular transformations and generalizations of several formulae of Ramanujan, The Rocky mountain J. Math. 7, no 1 (1977), 147-189.

[4] B. C. Berndt, Analytic Eisenstein series, theta-functions, and series relations in the spirit of Ramanujan, J. Reine. Angew. Math. 304 (1978), 332-365.

[5] M. Katsurada and T. Noda, Differential actions on the asymptotic expansions of non-holomorphic Eienstein series, to appear.

[6] N. N. Lebedev, Special functions and their applications, Dover Publications, Inc., New York, 1972.

[7] S. Lim, Generalized Eisenstein series and several modular transformation formulae, Ramanujan J. 19 (2009), 121-136.

[8] S. Lim, Modular transformation formulae for non-holomorphic Eisenstein series, to appear.

[9] P. Pasles and W. Pribitkin, A generalization of the Lipschitz summation formula and applications, Proc. Ams. Math. Soc. 129, no 11 (2001), 3177-3184. 
[10] L. J. Slater, Confluent hypergeometric functions, Cambridge University Press, 1960.

[11] E. C. Titchmarsh, Theory of functions, Oxford University Press, 1952.

Department of Mathematics

Mokwon University

Daejeon 302-318, Republic of Korea

E-mail: sglimj@mokwon.ac.kr 